\title{
A Unified Model of Drain Current Local Variability due to Channel Length Fluctuation for an $\mathrm{n}$-Channel EXDC MOS Transistor
}

\section{Sarmista Sengupta ( $\nabla$ sarmista.sengupta@gmail.com )}

Techno India Group

Soumya Pandit

Institute of Radiophysics and Electronics

\section{Original Research}

Keywords: Epitaxial delta doped channel, line edge roughness, channel length fluctuation, propagation of variance, roughness amplitude, correlation length

Posted Date: February 4th, 2021

DOI: https://doi.org/10.21203/rs.3.rs-173285/v1

License: (c) (i) This work is licensed under a Creative Commons Attribution 4.0 International License.

Read Full License

Version of Record: A version of this preprint was published at Silicon on July 31 st, 2021. See the published version at https://doi.org/10.1007/s12633-021-01218-w. 


\title{
A Unified Model of Drain Current Local Variability due to Channel Length Fluctuation for an n-channel E $\delta$ DC MOS Transistor
}

\author{
Sarmista Sengupta - Soumya Pandit
}

Received: date / Accepted: date

\begin{abstract}
A drain current local variability compact model due to random fluctuation of channel length induced by line edge roughness/line width roughness (LER/LWR) is derived here. The random fluctuation of channel length leads to correlated fluctuations of threshold voltage and effective mobility of the current carriers. Therefore, an unified compact model is required to combine all the causes. Our model is based on the principle of propagation of variance. For the model verification purpose, calibrated technology computer aided design (TCAD) simulation platform is extensively used for all possible bias regions and several LER profile parameters. Channel profile optimization is critically studied aiming reduction of $I_{D}$ variability.

The model is further extended for SOI (Silicon-on-insulator) transistor and validated with literature data of threshold voltage and on-current variability.
\end{abstract}

Keywords Epitaxial delta doped channel · line edge roughness · channel length fluctuation $\cdot$ propagation of variance $\cdot$ roughness amplitude $\cdot$ correlation length.

\section{Introduction}

In nano-scale CMOS analog circuits, mismatch between the drain currents of two identical transistors placed adjacent to each other is attributed to few

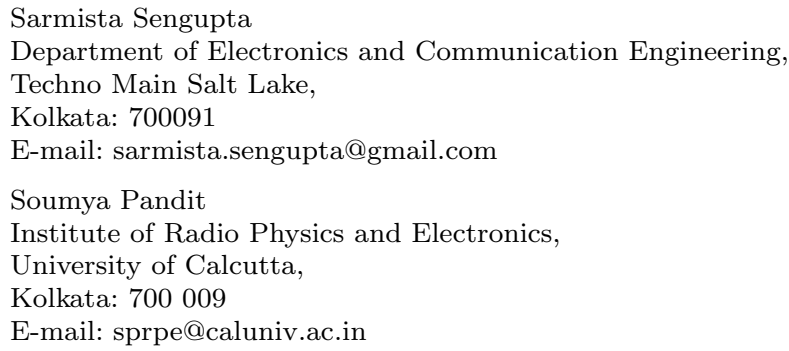


major causes like random discrete dopant effect (RDD), line edge/line width roughness (LER/LWR), metal gate granularity etc [1]. The line edge roughness (LER) phenomenon arises due to i) sub-wavelength lithography and/or ii) intrinsic non-uniformity of the photoresist used in the process technology [2]. LER leads to variation in critical dimension of the feature size. The amplitude of roughness $\left(\sigma_{L E R}\right)$ remains almost same and does not scale down with technology. Therefore, as the device dimensions, especially the channel length and width becomes comparable with this magnitude, LER/LWR-induced performance variability of a device/circuit appears to be quite critical [3].

\subsection{Literature Survey and Motivation}

In literature, the LER phenomenon has been studied primarily from two perspectives, i) its process level mitigation and ii) its impact on device performance. Several alternative patterning methods like extreme ultra violet lithography (EUVL), electron beam lithography (EBL), mask-less lithography, nanoimprint lithography etc. are being considered for enhanced resolution of the device dimension [4]. On the other hand, modeling and simulation of roughness and its impact on MOSFET, DGFET, FinFET etc. device performances have also been widely studied and reported. The variability impact of LER on sub $32 \mathrm{~nm}$ FinFET technologies is investigated from both device and circuit level perspectives using computer-aided design simulations in [5]. In [6] impact of LER along with other variability sources are reported for a Tunnel FET. A 3-D quasi-atomistic simulation methodology for LER in nonplanar devices, like FinFETs and gate-all-around (GAA) FETs, is proposed in [7]. The work reported in [8], describes the LER induced $V_{T}$ variability for $14 \mathrm{~nm}$ underlap FinFET using 3-D numerical simulations. For a better understanding of the performance variability induced by LER, modeling of the performance variability becomes indispensable. A tapered fin percolation model (TFPM) for evaluation of $V_{T}$ variability due to fin edge roughness (FER) of a FinFET is formulated in [9]. The model is based on a number of stochastically generated tapered fin structure with a minimum, maximum and average fin width. It can accurately capture the impact of associated physical parameters on $V_{T}$ variability. The model is extended for the formulation of drain current variability due to FER for a FinFET in [10]. On-current, off-current and sub-threshold slope variability due to FER are reported as well. Another model for $V_{T}$ variability formulated for a double gate MOSFET is reported in [11]. Unlike the previous one this model is physics-based and premises on the solution of $2 \mathrm{D}$ Poisson's equation. Here LER induced fluctuation occurs in the silicon body thickness. However neither of the above works give any compact model of $V_{T}$ or $I_{D}$ variability.

The major motivation behind undertaking the present research work is the lack of a suitable physics based compact drain current local variability model which incorporates the effect of line edge roughness on statistical fluctuation of the channel length. 
1.2 Outline and Contribution of our Work

An $E \delta D C$ transistor is a device structure proposed by us which is reported to be a low-power, low-cost transistor, suitable for SoC applications with controlled process variability effects due to random discrete dopant effects [12], [13], [14], [15], [16], [17]. In the present work, we derive a physics based local drain current variability model of an epitaxial delta doped channel MOS $(E \delta D C)$ transistor, caused due to random fluctuation of channel length, attributed to the LER/LWR phenomenon. The theoretical formulation of the model is based on the principle of propagation of variance. Assuming small fluctuation, first order Taylor's series is considered. The process varying parameters are i) channel length, ii) threshold voltage $\left(V_{T}\right)$, and iii) effective mobility. $V_{T}$ variability is induced due to channel length fluctuation and effective mobility varies due to fluctuating velocity saturation effect. The model predicted results are verified through calibrated technology computer aided design (TCAD) simulation platform. The model is verified for all possible bias regions and several LER profile parameters. Channel profile optimization is critically studied aiming reduction of drain current variability. The model is further extended for SOI (Silicon-on-insulator) transistor and validated with literature data of threshold voltage and on-current variability.

The local drain current variability is high when the transistor operates in the weak inversion mode (WI), compared to when operates in the strong inversion (SI) mode. In WI mode, local drain current variability depends on two factors, i) on channel length variability itself and ii) correlated $V_{T}$ variability. In SI mode, correlated effective mobility fluctuation component is also present apart from the components present in WI mode. As velocity saturation effect is more pronounced at high drain bias it contributes to the increased drain current variability at high drain bias apart from $V_{T}$ variability component.

The major salient features of our variability compact model are: (i) The formulation of the model is based upon the principle of propagation of variance, which is a formal theory of mathematical statistics. Our model encapsulates the device architecture through the use of appropriate compact model of the device architecture. By re-definitions of few device parameters, the model may thus easily be extended to other device structures such as SOI structures, which we have shown in this work. (ii) The present model of local drain current variability being a compact model gives an insight on the minimization approaches of variability through device design and optimization. (iii) The model does not involve purely empirical parameters.

\subsection{Organization of the Work}

The model of the $\sigma_{I_{D}}$ is derived in Section II, where we recall $\sigma_{V_{T}}$ formulation from previous work in brief. The verification the model with calibrated TCAD simulation results and the approaches for reduction of $\sigma_{I_{D}}$ is discussed in Section III. The extension of the model for SOI transistors and its validation with 


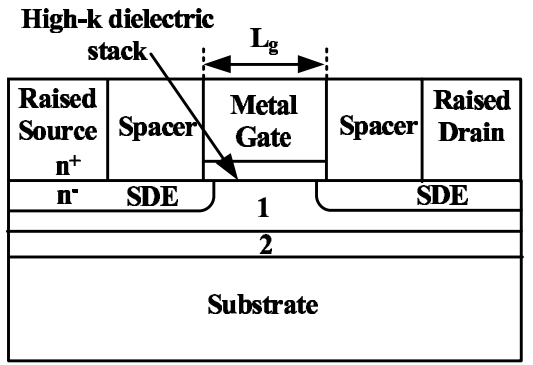

1: Undoped/Lightly doped epitaxial layer 2: High doped layer

Fig. 1 Schematic diagram of an Epitaxial $\delta$ doped n-channel MOS transistor used for design purpose.

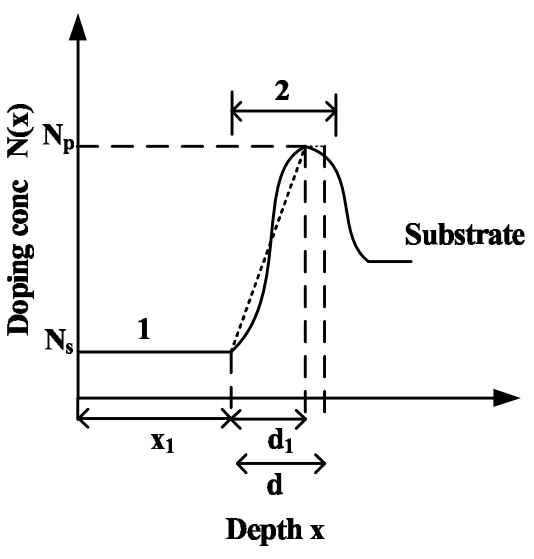

Fig. 2 Graded retrograde approximation of the channel profile of $\mathrm{E} \delta \mathrm{DC}$ transistor.

reported data are also mentioned in Section IV. Finally Section V concludes the paper.

\section{Theoretical Formulation of the Model}

\subsection{Background Information}

The cross-section and channel doping profile of the n-channel E $\delta$ DC transistor are as shown in Fig. 1 and Fig. 2. The significance of the various symbols used to represent the channel doping profile are as used in [18].

The threshold voltage for a short channel transistor, in the presence of the applied drain bias is written as [19],

$$
V_{T}=V_{T l}-\frac{2\left(V_{b i}-\psi_{S}\right)+V_{D S}}{2 \cosh \left(\frac{L}{2 l_{t}}\right)-2}
$$

where $V_{T l}$ is the long channel threshold voltage, $V_{b i}$ is the built-in potential across the $\mathrm{S} / \mathrm{D}$ junctions, $\psi_{s}=2 \Phi_{F}$ is the surface potential at strong inversion and $l_{t}$ is the characteristic length.

Using the principle of propagation of variance, random $V_{T}$ variability due to channel length fluctuation is given by [18],

$$
\sigma_{V_{T}}=\sqrt{\left(\frac{\partial V_{T}}{\partial L}\right)^{2}} \sigma L
$$

where threshold voltage sensitivity w.r.t. channel length is obtained using (1) as follows. 


$$
\frac{\partial V_{T}}{\partial L}=\frac{2\left(V_{b i}-\psi_{S}\right)+V_{D S}}{4 l_{t}\left[\cosh \left(\frac{L}{2 l_{t}}\right)-1\right]^{2}} \sinh \frac{L}{2 l_{t}}
$$

Due to the presence of LER/LWR, the average channel length of a transistor, becomes a random variable. $\sigma_{L}$ represents the variability of average channel of a transistor. Line edge roughness, and hence, $\sigma_{L}$ induced by LER is defined by correlation length $\Lambda$ and the rms roughness amplitude $\sigma_{L E R}$, as follows [20], [21], [22]. Here we assume uncorrelated edge variation, which gives channel length variability as follows [6], [23],

$$
\sigma_{L}=\frac{\sigma_{L W R}}{\sqrt{1+0.75 \frac{W}{\Lambda}}}=\sqrt{\frac{2}{1+0.75 \frac{W}{\Lambda}}} \sigma_{L E R}
$$

\subsection{Formulation of Drain Current Variability}

The drain current $I_{D}$ of a MOS transistor is related to several process parameters, which is mathematically represented by

$$
I_{D}=f(\bar{P})
$$

where $\bar{P}$ represents the vector of process parameters. Also we assume that the deviation/fluctuation in a certain parameter, symbolized as $\delta P_{i}$ is much smaller than the value of the parameter $P_{i}$ itself. Therefore, we employ first order Taylor's series expansion to write

$$
\frac{\delta I_{D}}{I_{D}} \approx \frac{1}{I_{D}} \frac{\partial I_{D}}{\partial P_{1}} \cdot \delta P_{1}+\frac{1}{I_{D}} \frac{\partial I_{D}}{\partial P_{2}} \cdot \delta P_{2}+\ldots . .
$$

The distribution of drain current samples for large number may be approximated to be a normal distribution, characterized by sample mean and standard deviation. The dispersion characteristics being more important in characterizing the variability aspect, we work with this parameter in our present work. From (6) the variance for three input parameters is written as

$$
\begin{array}{r}
\frac{\sigma_{I_{D}}^{2}}{I_{D}^{2}}=\left(\frac{1}{I_{D}} \frac{\partial I_{D}}{\partial P_{1}}\right)^{2} \cdot \sigma_{P_{1}}^{2}+\left(\frac{1}{I_{D}} \frac{\partial I_{D}}{\partial P_{2}}\right)^{2} \cdot \sigma_{P_{2}}^{2}+ \\
\left(\frac{1}{I_{D}} \frac{\partial I_{D}}{\partial P_{3}}\right)^{2} \cdot \sigma_{P_{3}}^{2}+\frac{2}{I_{D}^{2}} \frac{\partial I_{D}}{\partial P_{1}} \frac{\partial I_{D}}{\partial P_{2}} \cdot \rho_{12}\left(P_{1}, P_{2}\right) \cdot \sigma_{P_{1}} \cdot \sigma_{P_{2}}+ \\
\frac{2}{I_{D}^{2}} \frac{\partial I_{D}}{\partial P_{2}} \frac{\partial I_{D}}{\partial P_{3}} \cdot \rho_{23}\left(P_{2}, P_{3}\right) \cdot \sigma_{P_{2}} \cdot \sigma_{P_{3}}+ \\
\frac{2}{I_{D}^{2}} \frac{\partial I_{D}}{\partial P_{3}} \frac{\partial I_{D}}{\partial P_{1}} \cdot \rho_{31}\left(P_{3}, P_{1}\right) \cdot \sigma_{P_{3}} \cdot \sigma_{P_{1}}
\end{array}
$$


Here the factor $\rho$ characterizes the correlation between the input parameters. For perfect correlation, which occurs when the parameters are analytically related, the value of the correlation coefficient may be assumed to be unity.

The choice of each of the process parameter $P_{i}$ is based upon physics of the transistor. The sensitivity of the drain current with respect to these process parameters may be evaluated either through simulation method or through analytical method. In our work, we prefer the later and select appropriate compact model to describe the relationship between the drain current and the chosen process parameters.

\subsection{Drain Current Fluctuation through Charge based Model}

EKV drain current model, which smoothly describes the device operation in all the modes of operation is used to analyze drain current variability induced by LER/LWR [24], [25]. The average value of drain current is given as,

$$
I_{D}=I_{S}\left(i_{f}-i_{r}\right)
$$

where, $I_{S}=2 n \mu_{e f f} C_{o x}^{\prime} \frac{W}{L} \phi_{t}^{2}$ is called the specific current, and $i_{f}$ and $i_{r}$ are normalized forward and reverse currents with the normalization factor as $I_{S} . \mathrm{n}, C_{o x}^{\prime}, \mu_{e f f}$ and $\phi_{t}$ are the slope of gate-to-body voltage versus surface potential graph, the gate oxide capacitance per unit area, the effective mobility and the thermal voltage respectively. $i_{f / r}$ is as follows.

$$
i_{f / r}=\left[\ln \left\{1+\exp \left(\frac{V_{P}-V_{S / D}}{2 \phi_{t}}\right)\right\}\right]^{2}
$$

Here $V_{P}$ is the pinch-off voltage, approximately written as,

$$
V_{P}=\frac{V_{G}-V_{T}}{n}
$$

Here, source being the reference terminal, $V_{S}=0$. Normalized fluctuation of drain current due to L fluctuation and correlated $V_{T}$ and $\mu_{e f f}$ fluctuations thus becomes,

$$
\begin{aligned}
\frac{\delta I_{D}}{I_{D}} & =\frac{1}{I_{D}} \frac{\partial I_{D}}{\partial \mu_{e f f}} \delta \mu_{e f f}+\frac{1}{I_{D}} \frac{\partial I_{D}}{\partial L} \delta L+\frac{1}{I_{D}} \frac{\partial I_{D}}{\partial V_{T}} \delta V_{T} \\
& =\frac{\delta \mu_{e f f}}{\mu_{e f f}}-\frac{\delta L}{L}+\frac{1}{i_{f}-i_{r}}\left(\frac{\partial i_{f}}{\partial V_{T}}-\frac{\partial i_{r}}{\partial V_{T}}\right) \delta V_{T}
\end{aligned}
$$

The forward and reverse current derivatives w.r.t. $V_{T}$ referred in (11) are evaluated as follows.

$$
\frac{\partial i_{f}}{\partial V_{T}}=\frac{\ln \left[1+\exp \left(\frac{V_{P}}{2 \phi_{t}}\right)\right]}{1+\exp \left(\frac{V_{P}}{2 \phi_{t}}\right)} \cdot \frac{1}{\phi_{t}} \frac{\partial V_{P}}{\partial V_{T}}
$$




$$
\left.\frac{\sigma_{I_{D}}^{2}}{I_{D}^{2}}\right|_{W I}=\frac{\sigma_{L}^{2}}{L^{2}}+\frac{1}{\left(i_{f}-i_{r}\right)^{2}}\left(\frac{\partial i_{f}}{\partial V_{T}}-\frac{\partial i_{r}}{\partial V_{T}}\right)^{2} \sigma_{V_{T}}^{2}-2 \frac{\sigma_{L}}{L} \frac{1}{\left(i_{f}-i_{r}\right)}\left(\frac{\partial i_{f}}{\partial V_{T}}-\frac{\partial i_{r}}{\partial V_{T}}\right) \sigma_{V_{T}}
$$

$$
\frac{\partial i_{r}}{\partial V_{T}}=\frac{\ln \left[1+\exp \left(\frac{V_{P}-V_{D S}}{2 \phi_{t}}\right)\right]}{1+\exp \left(\frac{V_{P}-V_{D S}}{2 \phi_{t}}\right)} \cdot \frac{1}{\phi_{t}} \frac{\partial V_{P}}{\partial V_{T}}
$$

where, $\frac{\partial V_{P}}{\partial V_{T}}=-\frac{1}{n}$. In (11) both $\mu_{\text {eff }}$ and $V_{T}$ fluctuation roots from channel length fluctuation. Hence they are correlated.

If we now investigate (11), we see that the second term is independent of the bias region. The third term consisting of interpolation function, takes the corresponding limiting forms in different bias regions. The first term, i.e., mobility fluctuation needs a close inspection w.r.t. the nature of inversion region.

\subsection{Drain Current Variability in Weak Inversion (WI) Mode}

Velocity saturation effect being insignificantly small, can be neglected here. Hence, $\mu_{\text {eff }}=\mu_{s}$, where, $\mu_{s}$ is the surface mobility. In WI mode, surface mobility is solely dependent on Coulomb scattering of the carriers. i) The ionized impurity atoms present in the channel and ii) the remote fixed charges existing inside the high-k dielectric (Remote Coulomb Scattering or RCS effect) act as the scattering centers. For low normal electric field, the inversion carriers experience surface mobility as follows [26]

$$
\frac{1}{\mu_{s}}=\frac{1}{\mu_{B}}+\frac{1}{\mu_{R C S}} \approx \frac{1}{\mu_{00}}+\alpha_{d} \cdot Q_{i m p}
$$

Here, RCS effect is embedded in $\mu_{00}$ and it is termed as the zero field mobility. Coulomb scattering with the impurity atoms is represented by the term $\alpha_{d} . Q_{i m p}, \alpha_{d}$ being the Coulomb scattering parameter. However, channel length of the transistor does not have any impact on the scattering phenomena. Hence surface mobility fluctuation term on R.H.S. of (11) vanishes.

$$
\frac{\delta \mu_{\mathrm{eff}}}{\mu_{\mathrm{eff}}}=\frac{\delta \mu_{s}}{\mu_{s}}=0
$$

Thus normalized variance of drain current in weak inversion mode is thus given by (16).

The last term in (16) is the correlation coefficient. The current sensitivities are calculated using (12) and (13). 


$$
\begin{aligned}
\left.\frac{\sigma_{I_{D}}^{2}}{I_{D}^{2}}\right|_{S I} & =\frac{\sigma_{\mu_{e f f}^{2}}}{\mu_{e f f}^{2}}+\frac{\sigma_{L}^{2}}{L^{2}}+\frac{1}{\left(i_{f}-i_{r}\right)^{2}}\left(\frac{\partial i_{f}}{\partial V_{T}}-\frac{\partial i_{r}}{\partial V_{T}}\right)^{2} \sigma_{V_{T}}^{2}-2 \frac{\sigma_{\mu_{e f f}}}{\mu_{e f f}} \frac{\sigma_{L}}{L} \\
& -2 \frac{\sigma_{L}}{L} \frac{1}{\left(i_{f}-i_{r}\right)}\left(\frac{\partial i_{f}}{\partial V_{T}}-\frac{\partial i_{r}}{\partial V_{T}}\right) \sigma_{V_{T}}+2 \frac{\sigma_{\mu_{e f f}}}{\mu_{e f f}} \frac{1}{\left(i_{f}-i_{r}\right)}\left(\frac{\partial i_{f}}{\partial V_{T}}-\frac{\partial i_{r}}{\partial V_{T}}\right) \sigma_{V_{T}}
\end{aligned}
$$

2.5 Drain Current Variability in Strong Inversion (SI) Mode

In strong inversion (SI) mode, The effective mobility is [25]

$$
\mu_{\mathrm{eff}}=\frac{\mu_{s}}{1+\frac{\mu_{s} V_{D S}}{L v_{\mathrm{sat}}}}
$$

here $\mu_{s}$ represents the surface carrier mobility and $v_{\text {sat }}$ represents the saturation velocity of the inversion carriers.

To evaluate fluctuation of $\mu_{e f f}$, in SI mode, we refer to (17), which on partial differentiation w.r.t. L, gives,

$$
\delta \mu_{e f f}=\mu_{e f f}^{2} \frac{V_{D S}}{v_{s a t} L^{2}} \delta L=\left(\frac{\mu_{s}}{1+\frac{\mu_{s} V_{D S}}{L v_{\text {sat }}}}\right)^{2} \cdot \frac{V_{D S}}{v_{s a t} L^{2}} \delta L
$$

In this mode of operation, surface roughness scattering and lattice vibration dominates surface mobility [27]. An efficient surface mobility model which includes the mobility degradation effect is as follows.

$$
\mu_{s}=\frac{\mu_{0}}{1+\theta_{1}\left(V_{G S}-V_{T}\right)+\theta_{2}\left(V_{G S}-V_{T}\right)^{2}}
$$

Here $\theta_{1}$ and $\theta_{2}$ are the parameters to account for the mobility degradation effect [28], [29]. $\mu_{0}$ represents low field mobility of the inversion carriers, which includes both the Coulomb scattering effects as mentioned in the previous section. In presence of the series source/drain (S/D) resistance mobility degradation parameter $\theta_{1}$ is modified as [28]

$$
\theta_{1}=\theta_{1}^{*}+\frac{C_{o x} R_{S D} W \mu_{0}}{L}
$$

Here, $\theta_{1}^{*}$ is the coefficient of mobility degradation in absence of source/drain resistance and $R_{S D}$ is the source/drain series resistance. The values of the mobility degradation coefficients are extracted from extensive TCAD simulation.

The normalized drain current variability due to channel length fluctuation in SI mode is thus given by (21).

The last three terms in (21) represent the corresponding correlation coefficients. The current sensitivities are calculated using (12) and (13). Effective mobility variability is derived using (18) as,

$$
\frac{\sigma_{\mu_{e f f}^{2}}}{\mu_{e f f}^{2}}=\left(\frac{\mu_{s}}{1+\frac{\mu_{s} V_{D S}}{L v_{\mathrm{sat}}}}\right)^{2} \cdot\left(\frac{V_{D S}}{v_{\text {sat }} L^{2}}\right)^{2} \sigma_{L}^{2}
$$




\section{Model Implementation and Validation}

\subsection{Device Simulation}

The n-channel E $\delta$ DC MOS transistor parameters are selected for high performance applications according to the International Technology Roadmap for Semiconductors 2010 version. The models for different physical effects are included in the simulation as discussed in [13], [17]. The channel length varaibility due to LER is simulated using monte carlo simulation for 200 samples. The channel length of $200 \mathrm{E} \delta \mathrm{DC}$ transistors are normally distributed around its nominal value (here $16 \mathrm{~nm}$ ) with a spread of $\sigma_{L}$. The value of $\sigma_{L}$ is calculated for a particular set of $\Lambda$ and $\sigma_{L E R}$ values using 4 .

\subsection{Model Parameter Calculation}

The analytical model incorporates various empirical parameters like, $\mu_{0}, \theta_{1}$ and $\theta_{2}$. The extraction methods of these parameters are reported in [17]. Their extracted values are, $\mu_{0}=202 \mathrm{~cm}^{2} / V . s, \theta_{1}=0.845 / V$ and $\theta_{2}=2.07 / V^{2}$ following [30].

\subsection{Variation of Local Drain Current Variability with Bias}

As the transistor moves from weak inversion (WI) mode to strong inversion (SI) mode, the variability gradually decreases, as can be seen from Fig. 3 . This is due to the fact that the deterministic drain current $\left(I_{D}\right)$ is less by several orders in WI mode compared to SI mode. This leads to the nature of variation of normalized drain current variability even for similar fluctuation $\sigma I_{D}$ over the entire gate bias range.

As evident from (16) In the weak inversion mode (WI), the local drain current variability depends upon three factors: the channel length variability, the threshold voltage variability and the correlation factor. Since the channel length variability term is bias independent, the drain bias dependence comes from the remaining two components. Moreover, we observe that higher drain current variability is more pronounced in WI mode compared to strong inversion (SI) mode. Though $\sigma_{V_{T}}$ is independent of the bias mode (weak or strong), the higher drain current variability for high drain bias at WI mode, compared to SI mode results from exponential drain bias dependence of inversion charge at drain end in WI mode compared to its linear drain bias dependence in SI mode [25].

However, as we can see from (21), in SI mode, correlated effective mobility fluctuation component is also present apart from the components present in WI mode. As velocity saturation effect is more pronounced at high $V_{D S}$, it contributes to the increased drain current variability at high drain bias apart from $V_{T}$ variability component. An important point for $\mathrm{E} \delta \mathrm{DC}$ transistor may 


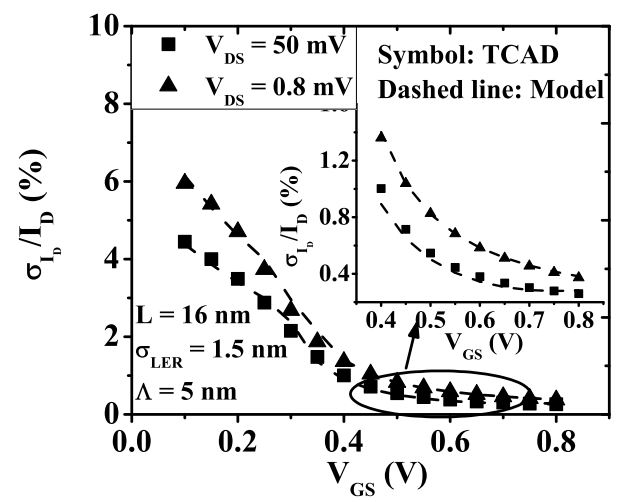

Fig. 3 Variation of the normalized drain current variability with $V_{G S}$ for $\mathrm{L}=16 \mathrm{~nm}$ at low and high drain biases.

be noted here. We reported in our earlier works [13], [17], that the threshold voltage variability at high drain bias is not much significant. The excellent agreement between TCAD simulation and analytical results confirms perfect capture of bias dependence of the model.

\subsection{Local Drain current Variability for different LER profile parameters}

In Fig. 4(a), the variation of normalized local drain current variability due to random variation of channel length is reported for low drain bias and for a range of $\sigma_{L E R}$ values. For this observation $\Lambda$ is kept fixed at $5 \mathrm{~nm}$. With increasing $\sigma_{L E R}$, channel length variability increases linearly following (4). Increased channel length variability induces increased $V_{T}$ variability and hence normalized drain current variability due to channel length variability enhances. A similar variation is reported in Fig. 4(b) for high value of drain-to-source voltage. As discussed in a previous subsection, we see here that nature of the graphs remaining similar to those at low $V_{D S}$, variability values are higher for high $V_{D S}$, which is a consequence of higher $\sigma_{V_{T}}$ for high $V_{D S}$.

The nature of graphs given in Fig. 4(a) and Fig. 4(b) are not only physically justified, but also this physics is perfectly incorporated in the model. This is evident from the very close agreement of the simulation and the model predicted results.

Fig. 5(a) represents the variation of normalized local drain current variability due to random variation of channel length for low drain bias and for a range of $\Lambda$ values. For this observation $\sigma_{L E R}$ is kept fixed at $1 \mathrm{~nm}$. With increasing $\Lambda$, channel length variability increases following (4). Increased channel length variability induces increased $V_{T}$ variability and drain current variability. However, this enhancement is significant in WI mode of operation. A similar variation is reported in Fig. 5(b) for high value of $V_{D S}$. Here also $\sigma_{I_{D}}$ values 


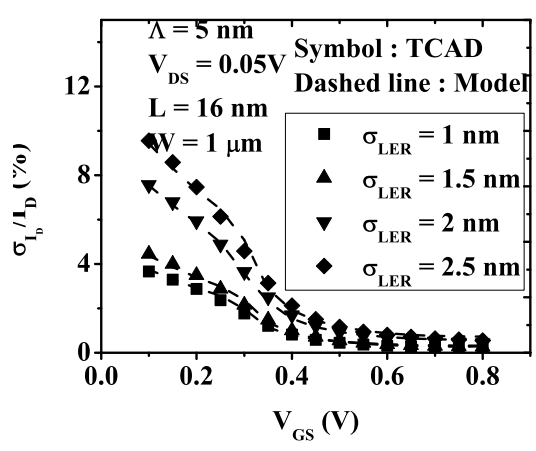

(a)

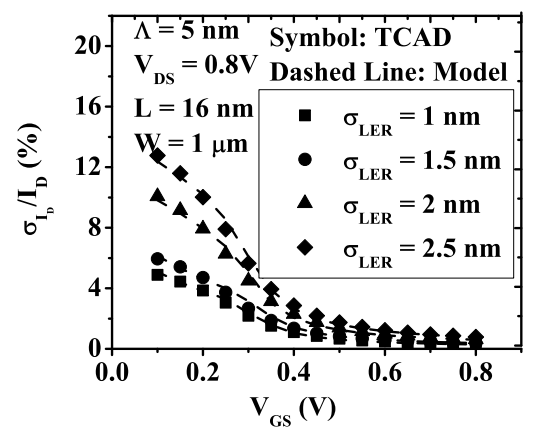

(b)

Fig. 4 Variation of normalized drain current variability with applied gate bias for various edge roughness amplitude at $L=16 \mathrm{~nm}$ at (a) Low $V_{D S}$ and (b) High $V_{D S}$.

are higher for high $V_{D S}$ as correlated $\sigma_{V_{T}}$ increases with increasing drain bias. Here the scalability of model is verified with LER correlation length $\Lambda$.

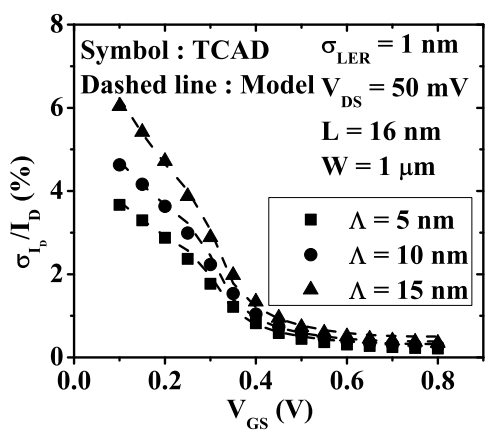

(a)

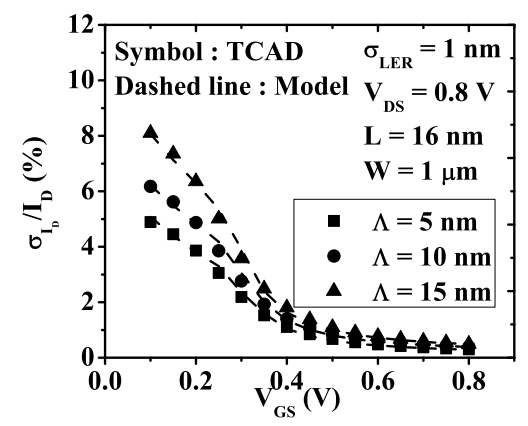

(b)

Fig. 5 Variation of normalized drain current variability with applied gate bias for various correlation lengths at $L=16 \mathrm{~nm}$ at (a) Low $V_{D S}$ and (b) High $V_{D S}$.

3.5 Reduction of Drain Current Local Variability through Channel Engineering

$\sigma_{I_{D}}$ due to variability of channel length induced by LER can be controlled in an E $\delta$ DC transistor using channel engineering technique. This technique involves the study of variability performance with variation in the profile parameters using analytical model. Here we make all the observations for high drain bias and for a fixed LER profile $\left(\Lambda=5 \mathrm{~nm} \& \sigma_{L E R}=3 \mathrm{~nm}\right)$, hence for a fixed channel 
length variability. If we have a close look at (16) and (21), profile parameter dependence of the normalized drain current variability is only associated with the $V_{T}$ variability component. In the following discussion we would refer to our observation obtained in [18].

\subsubsection{Variation with $x_{1}$}

In Fig. 6(a), we observe the variation of $\sigma_{I_{D}} / I_{D}$ with gate bias for several epitaxial layer thickness $x_{1}$. As we are studying this section for fixed channel length variability, correlated $V_{T}$ variability differs with change of profile parameter values. With increasing thickness of the low doped layer $x_{1}$, depletion depth, $W_{d m}$ increases. Increased $W_{d m}$, worsens short channel effect (SCE) [15]. Poor SCE is manifested by larger $V_{T}$. Hence $V_{T}$ will be more sensitive to variation in channel length, leading to higher $\frac{\partial V_{T}}{\partial L}$. Hence, $\sigma_{V_{T}}$ increases with increasing $x_{1}$. Consequently the contribution of $V_{T}$ variability associated component of normalized drain current varibility increases and hence $\sigma_{I_{D}} / I_{D}$ goes up. It is observed that as $x_{1}$ increases from $5 \mathrm{~nm}$ to $15 \mathrm{~nm}, \sigma_{I_{D}} / I_{D}$ becomes three times larger for $V_{G S}=0.1 \mathrm{~V}$. However this increment lowers as the transistor approaches SI mode of operation.

\subsubsection{Variation with $N_{s}$}

Fig. 6(b) shows the variation of $\sigma_{I_{D}} / I_{D}$ with gate bias for several epitaxial layer doping $N_{s}$. With increase of epitaxial layer doping, the short channel effect is controlled, hence the maximum depletion depth and consequently $\sigma_{V_{T}}$ is restricted. Lowering of $\sigma_{V_{T}}$ in-turn lowers $\sigma_{I_{D}} / I_{D}$ with increasing $N_{s}$. The maximum improvement of $\sigma_{I_{D}} / I_{D}$ is by $8.5 \%$ which occurs for $V_{G S}=0.1 \mathrm{~V}$. The improvement gradually diminishes with increase of gate bias and becomes insignificant as the transistor enters in SI mode.

\subsubsection{Variation with $N_{p}$}

Fig. 6(c) shows the variation of $\sigma_{I_{D}} / \bar{I}_{D}$ with gate bias for several screening layer doping $N_{p}$. Increase in $N_{p}$ restricts the spread of depletion region beneath the channel. Controlled channel depletion makes SCE better. Hence $V_{T}$ variability and drain current variability with random variation of channel length is reduced with increasing $N_{p}$. However, minimum value of channel depletion is limited by $x_{1}$, SCE and hence variability due to random variation of channel length cannot be reduced indefinitely. Consequently, we see in Fig. 6(c), $\sigma_{I_{D}} / I_{D}$ does not reduce significantly, for $N_{p} \geq 5 \times 10^{19} \mathrm{~cm}^{-3}$.

In the above observations we find that the impact of all the three profile parameters on drain current variability becomes insignificant as we enter in SI mode. This is because, in SI mode correlated effective mobility fluctuation plays a key role which is independent of channel profile. 


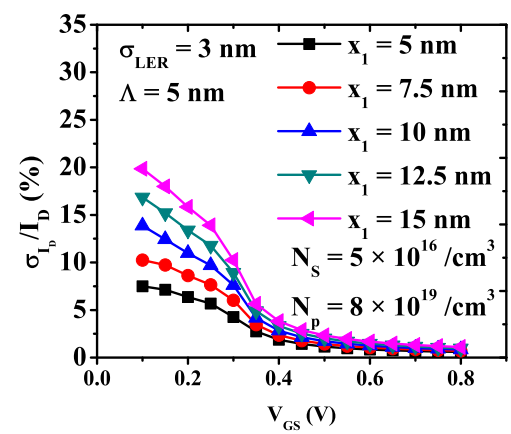

(a)

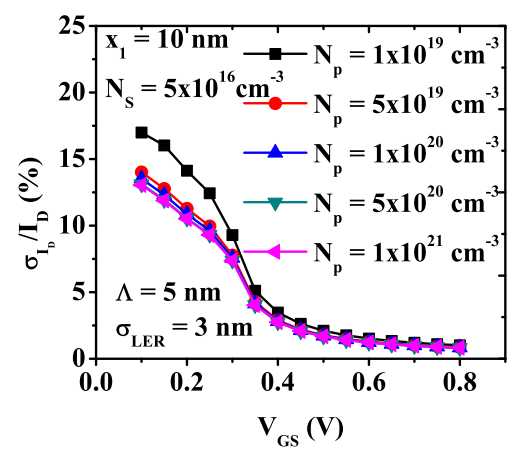

(c)

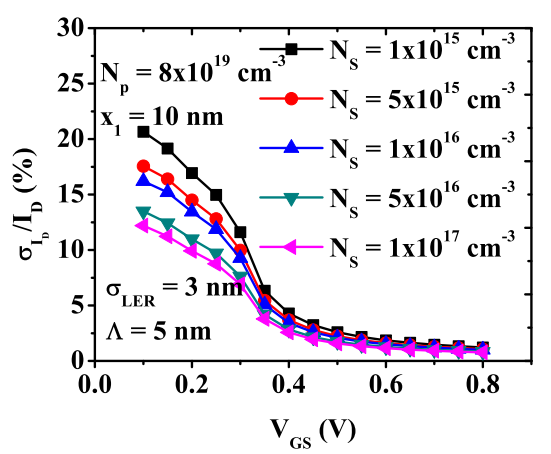

(b)

Fig. 6 Effect of channel profile parameters on normalized drain current variability for different values of (a) $x_{1}$, (b) $N_{s}$ and (c) $N_{p}$

\section{Application of the model for FD-SOI Transistor}

The model derived in the present work, can be extended for UTB-FD-SOI MOSFET, with the definition of depletion depth as described in [31]. The results obtained from the extended model for SOI structure are compared with the reported results [32] of atomistic simulation results for $V_{T}$ variability and local ON current variability due to LER. The comparison is made for all three reported structures with different gate-oxide and body thickness. LER parameters are $3 \sigma_{L E R}=2 \mathrm{~nm}$ and $\Lambda=25 \mathrm{~nm}$. The model parameters are suitably selected to match the corresponding nominal performances. The validity of the extended model is depicted in Fig. 7, where appreciable accuracy is observed for both $V_{T}$ and $I_{O N}$ variability. Hence the extended model is verified for the SOI structures. 


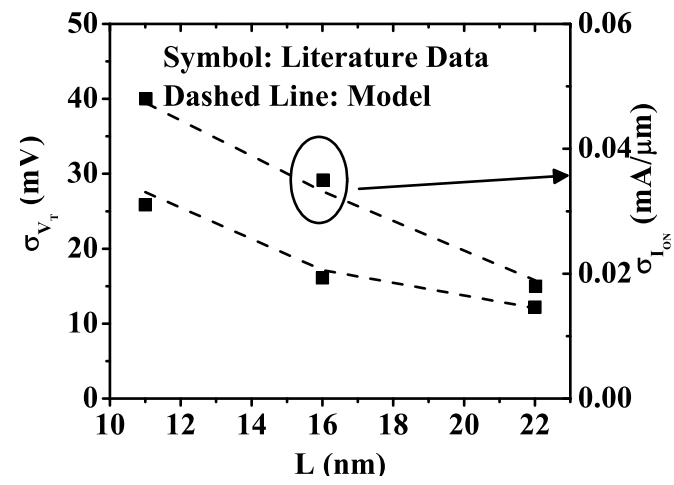

Fig. 7 Verification of the model predicted local ON-current variability result due to LER with atomistic simulation results for a UTB-FD-SOI MOSFET [32].

\section{Conclusion}

An unified model of drain current local variability due to channel length fluctuation caused by LER phenomenon is reported. The channel length variation leads to correlated variation of threshold voltage and effective mobility of the charge carriers. The drain current variability is significant in weak inversion mode compared to strong inversion mode. The variability is higher for higher drain bias (due to the presence of DIBL) irrespective of mode of operation. In the strong inversion mode, the correlated fluctuation due to effective mobility of the carriers plays an important role. Validation of the model is established by the close agreement between the model predicted results and those obtained from calibrated TCAD results. Further, extensive channel engineering approach prescribes the way for designing an optimal channel profile for the $\mathrm{E} \delta \mathrm{DC}$ transistor with minimum $V_{T}$ and $I_{D}$ local variability due to channel length fluctuation.

Fine tuning of the correlation components may be included in the model by introducing a general correlation parameter, value of which may be determined from a set of measured data. We have shown the extension of our model to SOI structure. With appropriate use of compact model of nominal drain current and redefinition of some parameters, the model may be extended to tri-gate devices also.

\section{Conflict of interest}

The authors declare that they have no conflict of interest. 


\section{Author contributions}

Both the authors contributed to the conception and formulation of the model. Calibration of the simulator, simulation, model formulation, processing and simulation of the simulated data were done by the corresponding author. Problem formulation, model development and its physical analysis was performed by the second author. The first draft of the manuscript was written by the corresponding author and was critically checked and modified by the second author. Both the authors read and approved the final manuscript.

\section{Availability of data and material}

Not Applicable

\section{Compliance with ethical standards}

Not Applicable

\section{Consent to participate}

Not Applicable

\section{Consent for Publication}

Not Applicable

\section{Acknowledgments}

The second author thanks Department of Electronics and Information Technology, Govt. of India for utilizing the resources obtained under the SMDP-C2SD project at the University of Calcutta.

\section{References}

1. S. Pandit, C. Mandal, A. Patra, Nano-Scale CMOS Analog Circuits Models and CAD Techniques for High-Level Design (CRC Press, 2014)

2. C. Shin, Variation-Aware Advanced CMOS Devices and SRAM (Springer Series in Advanced Microelectronics 56, Springer Science+Business Media Dordrecht, 2016)

3. D. Reid, S. Millar, C. Roy, A. Asenov, IEEE Transactions on Electron Devices 57(11), $2801(2010)$

4. R.M.M. Hasa, X. Luo, Nanomanufacturing and Metrology 1, 67 (2018)

5. G. Leung, L. Lai, P. Gupta, C.O. Chui, IEEE Transactions on Electron Devices 59, 2057 (2012) 
6. G. Leung, C.O.a. Chui, IEEE Transactions on Electron Devices 60, 84 (2013)

7. S. Oh, C. Shin, IEEE Transactions on Electron Devices 63(12), 4617 (2016)

8. R.S. Rathore, R. Sharma, A.K. Rana, Superlattices and Microstructures 103, 304 (2017)

9. S. Mittal, A.S. Shekhawat, U. Ganguly, IEEE Trans Electron Devices 63(3), 1352 (2016)

10. S. Mittal, Amita, A.S. Shekhawat, S. Ganguly, U. Ganguly, IEEE Trans Electron Devices 64(8), 3489 (2017)

11. B. Sriram, S.R.and Bindu, Journal of Computational Electronics 19, 622 (2020)

12. A. Asenov, S. Saini, IEEE Trans. Electron Devices Vol.46, 1718 (1999)

13. S. Sengupta, S. Pandit, IEEE Trans. on Electron Devices 63(2), 551 (2016). DOI:10.1109/TED.2015.2507065

14. R. Das, A.K. Gond, S. Sengupta, R.R. Sahani, S. Pandit, Microsystem Technologies DOI 10.1007/s00542-016-2995-z, 1 (2016)

15. S. Sengupta, S. Pandit, International Journal of Electronics Vol. 102, No. 6, 967 (2014). DOI: $10.1080 / 00207217.2014 .945194$

16. S. Sengupta, S. Sikdar, S. Pandit, International Journal of Electronics (2016). DOI 10.1080/00207217.2016.1180546. DOI: 10.1080/00207217.2016.1180546

17. S. Sengupta, S. Pandit, IEEE Transactions on Electron Devices 65(4), 1267 (2018)

18. S. Sengupta, S.and Pandit, in Proceedings of Devices for Integrated Circuit (DevIC). (IEEE, 2017), pp. 685-689

19. Z. Liu, etal, IEEE Trans. Electron Devices Vol.40, pp.86 (1993)

20. A. Asenov, S. Kaya, A.R. Brown, IEEE Transactions on Electron Devices 50, 1254 (2003)

21. Y.M.e.a. Sabry, Toward Quantum FinFET (Springer, 2013)

22. G. Leung, C.O. Chui, IEEE Electon Device Letters 32, 1489 (2011)

23. N. Seoane, G. Indalecio, E. Comesana, M. Aldegunde, A.J. Gracia-Loureiro, K. Kalna, IEEE Transactions on Electron Devices 61, 466 (2014)

24. E.A. Vittoz, C.C. Enz, Sub-threshold Design for Ultra Low-Power Systems. Series on Integrated Circuits and Systems. (Springer, Boston, MA, 2006), chap. EKV Model of the MOS Transistor, pp. 49-74

25. A.B.Bhattacharyya, Compact MOSFET Models for VLSI Design (John Wiley and Sons (Asia) Pte Ltd, 2009). DOI: 10.1002/9780470823446

26. A. Emrani, F. Balestra, G. Ghibaudo, Solid State Electronics 37, 1723 (1994). DOI: 10.1016/0038-1101(94)90219-4

27. M. Mezzomo, C, A. Bajolet, G. Cathignol, A. Ghinbaudo, Solid State Electronics Vol. 65-66, 163 (Novermber-December 2011). DOI: 10.1016/j.sse.2011.06.040

28. C. Nguyen-Duc, S. Cristoloveanu, G. Ghibaudo, Solid State Electronics 29(12), 1271 (1986). DOI: 10.1016/0038-1101(86)90133-4

29. N. Arora, MOSFET Modeling for VLSI Simulation: Theory and Practice (World Scientific, 2006). DOI: 10.1142/9789812707581

30. P.K. McLarty, S. Cristoloveanu, O. Faynot, V. Misra, J.R. Hauser, J.J. Wortman, Solid State Electronics 38, 1175 (1995). DOI: 10.1016/0038-1101(94)00248-E

31. N. Sugii, R. Tsuchiya, T. Ishigaki, Y. Morita, H. Yoshimoto, S. Kimura, IEEE Transactions Electron Devices 57, 835 (2010). DOI: 10.1109/TED.2010.2040664

32. S. Markov, A. Zain, B. Cheng, A. Asenov, in SOI Conference (2012). DOI: 10.1109/SOI.2012.6404365 
Figures

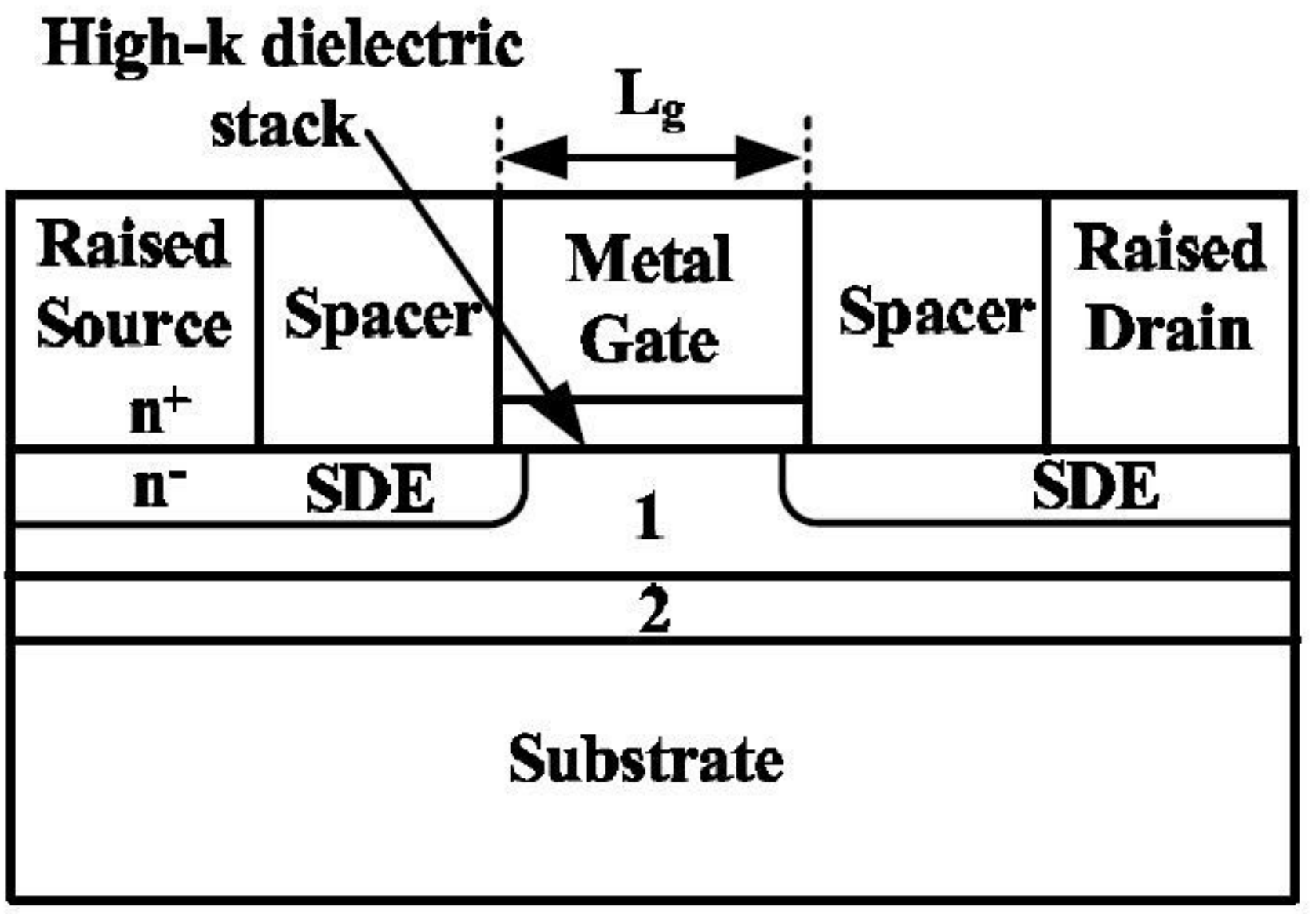

\section{1: Undoped/Lightly doped epitaxial layer 2: High doped layer}

Figure 1

Schematic diagram of an Epitaxial $\delta$ doped n-channel MOS transistor used for design purpose. 


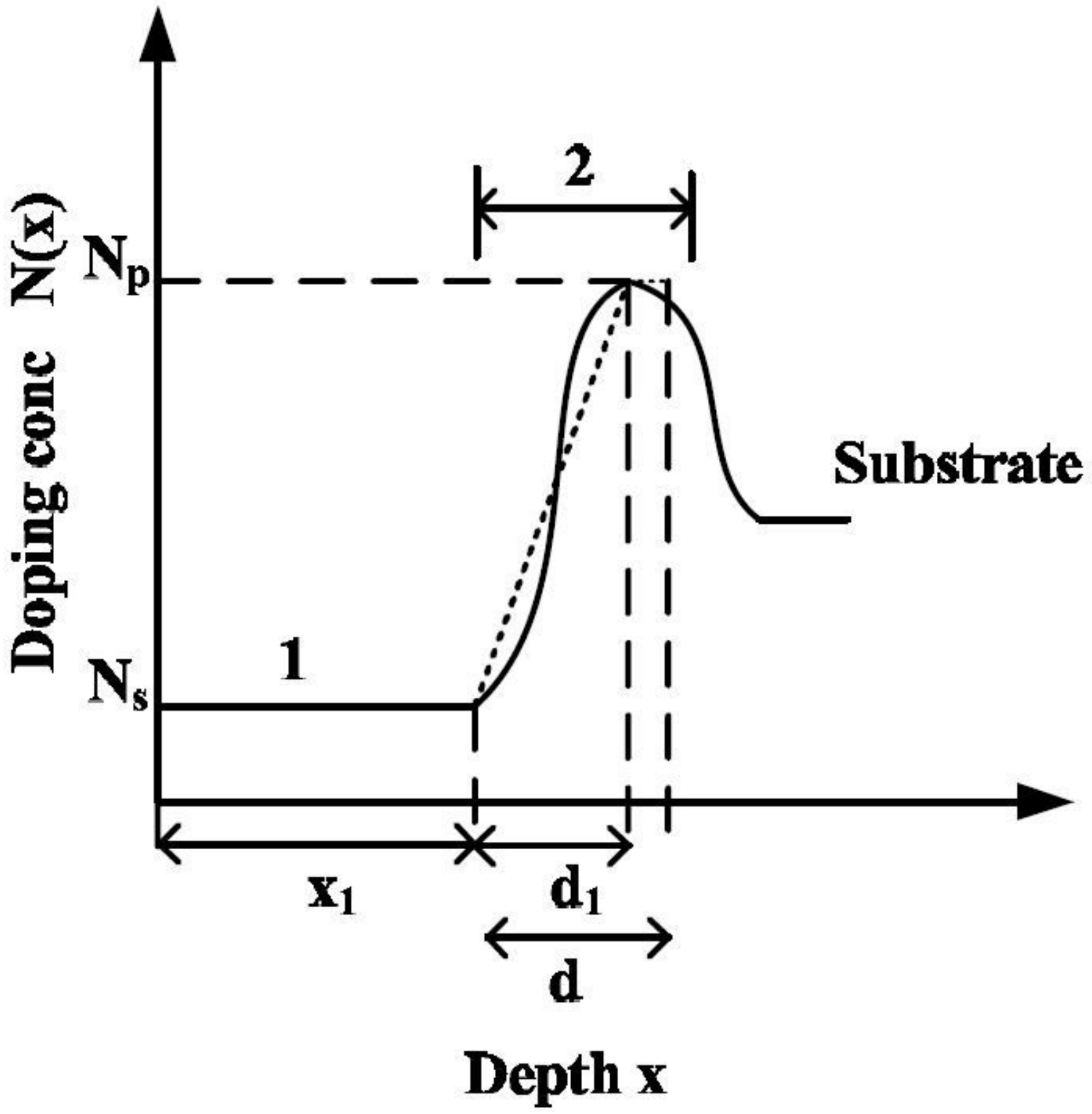

Figure 2

Graded retrograde approximation of the channel profile of ESDC transistor. 


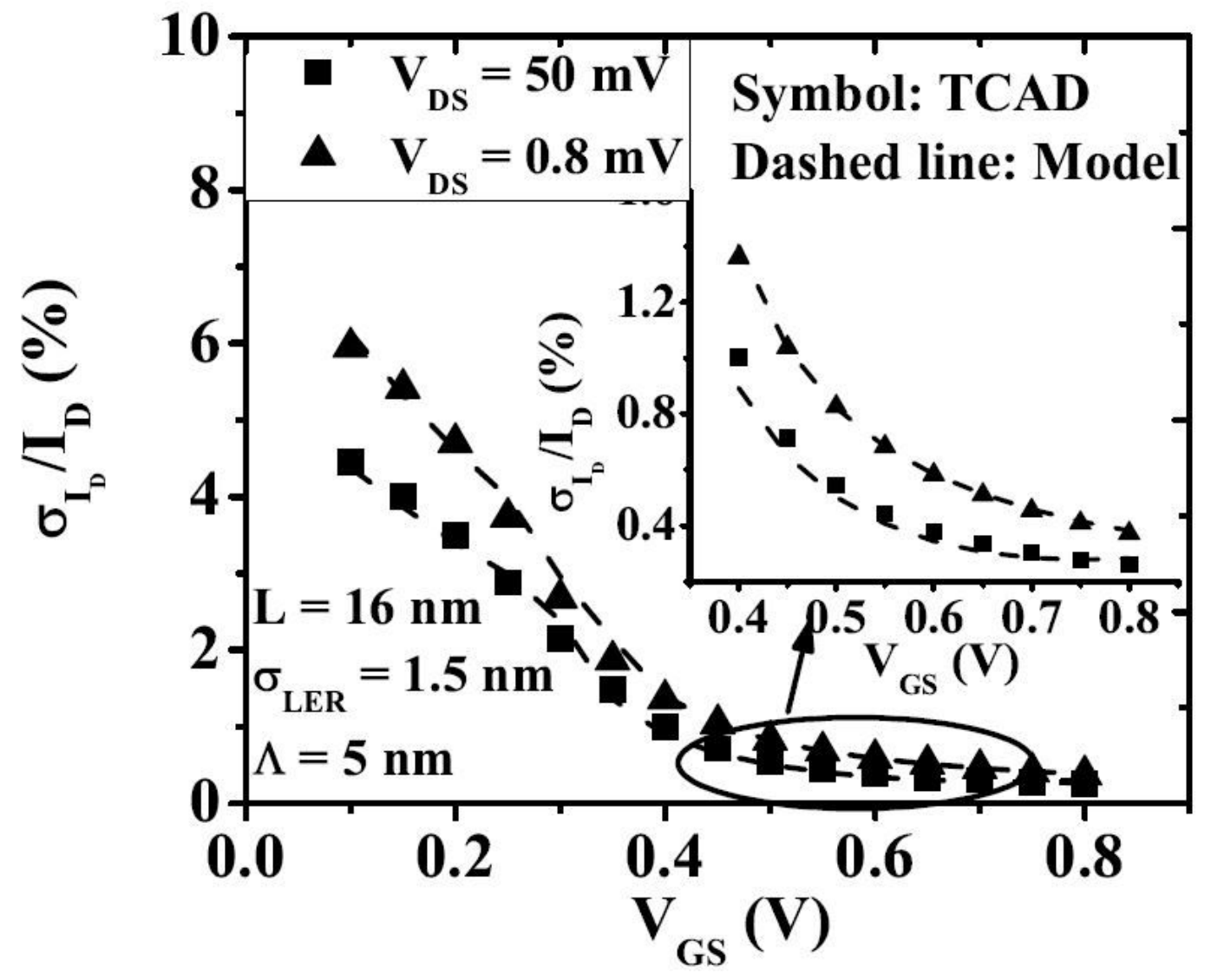

Figure 3

Variation of the normalized drain current variability with VGS for $\mathrm{L}=16 \mathrm{~nm}$ at low and high drain biases.

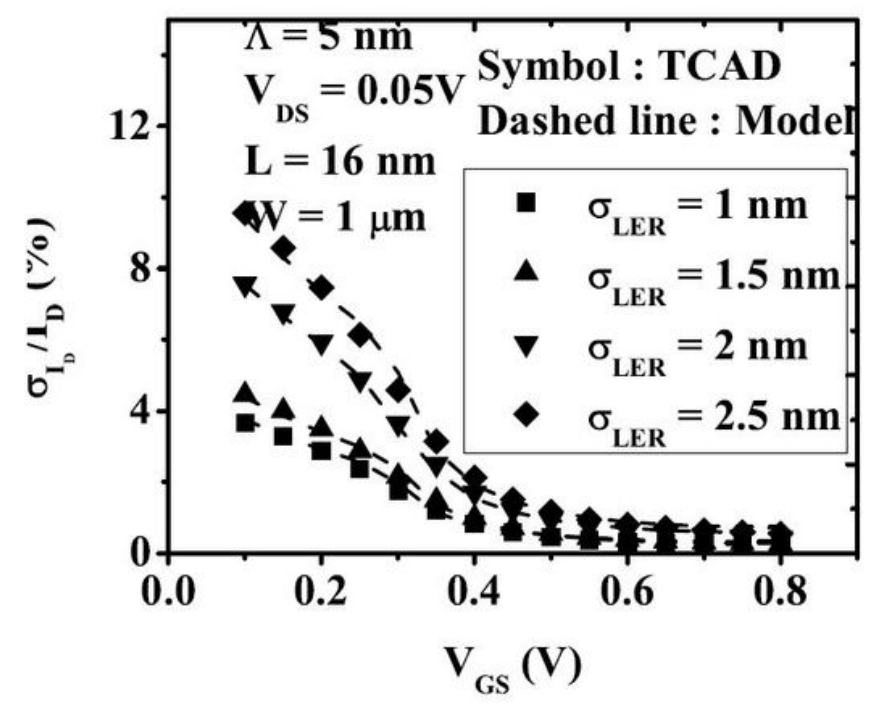

(a)

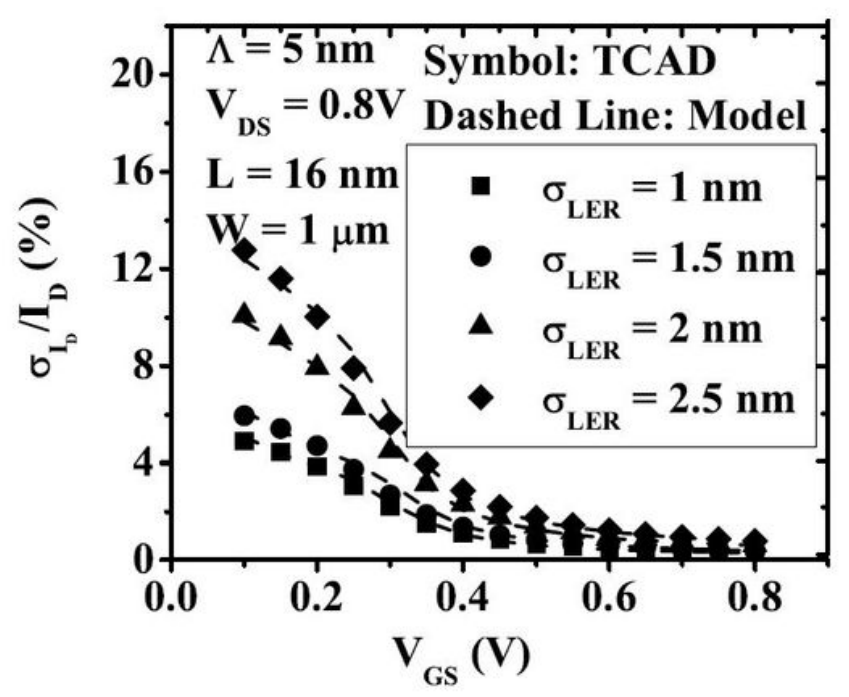

(b)

Figure 4 
Variation of normalized drain current variability with applied gate bias for various edge roughness amplitude at $\mathrm{L}=16 \mathrm{~nm}$ at (a) Low VDS and (b) High VDS.

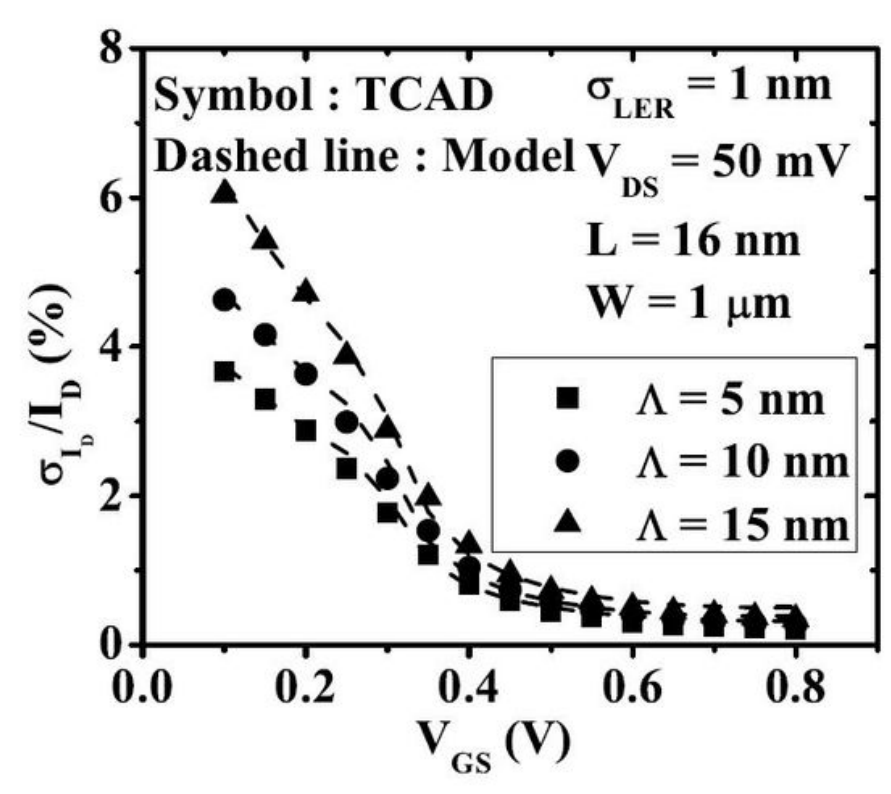

(a)

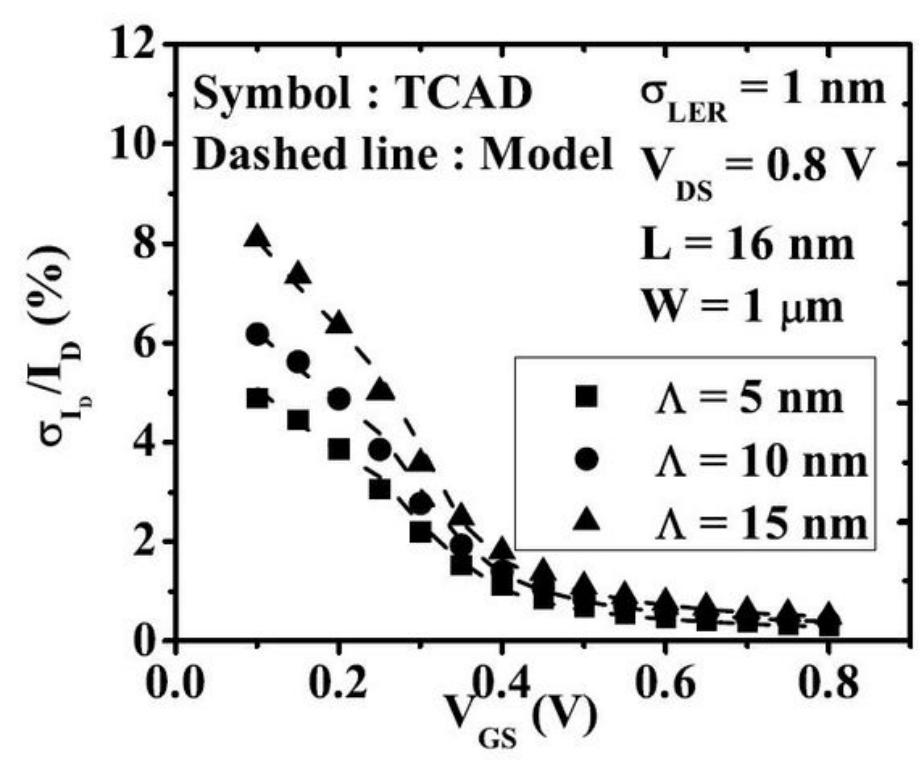

(b)

Figure 5

Variation of normalized drain current variability with applied gate bias for various correlation lengths at $\mathrm{L}$ $=16 \mathrm{~nm}$ at $(\mathrm{a})$ Low VDS and (b) High VDS. 


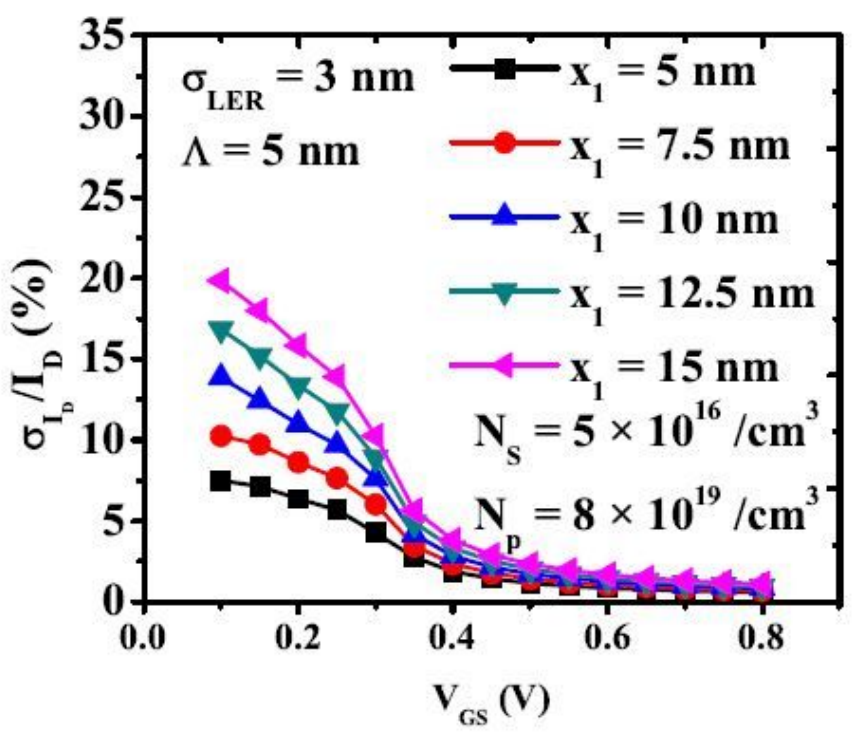

(a)

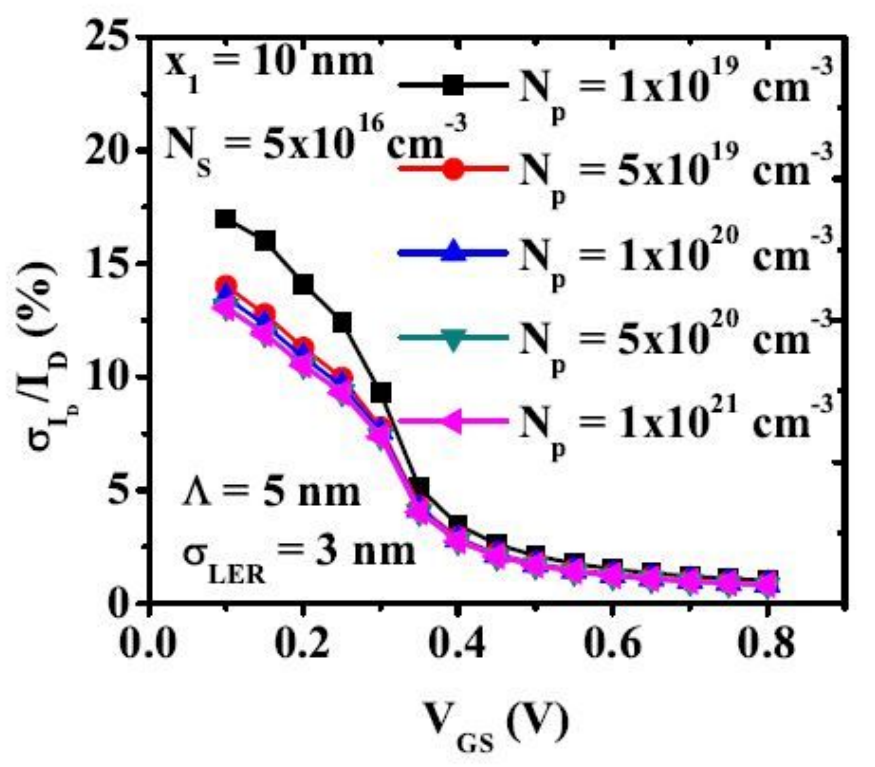

(c)

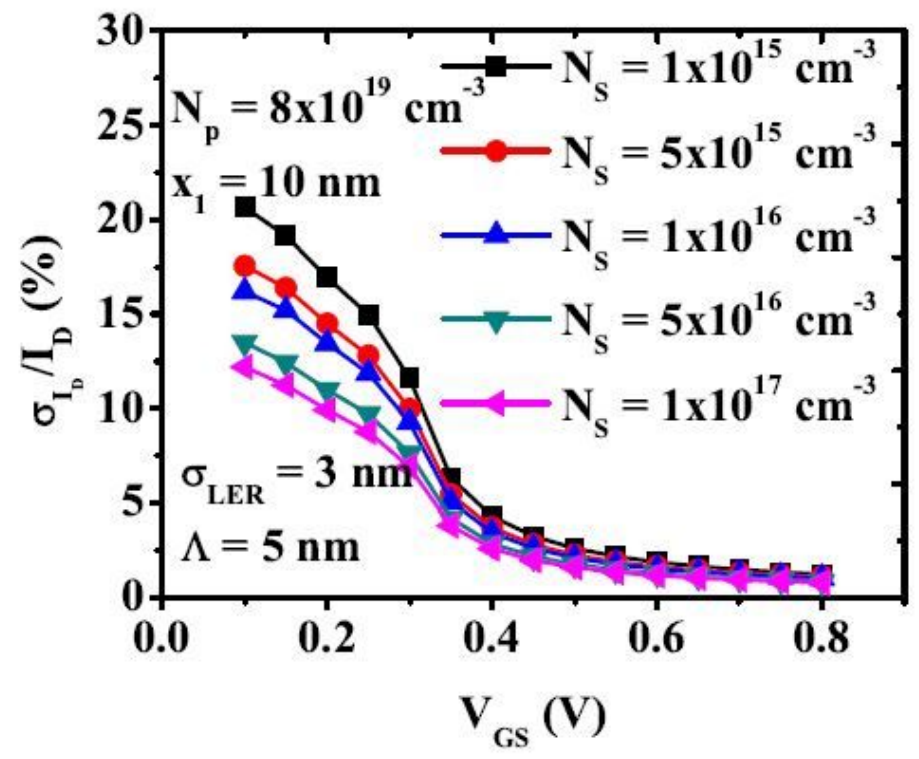

(b)

Figure 6

Effect of channel profile parameters on normalized drain current variability for different values of (a) $x 1$, (b) Ns and (c) Np 


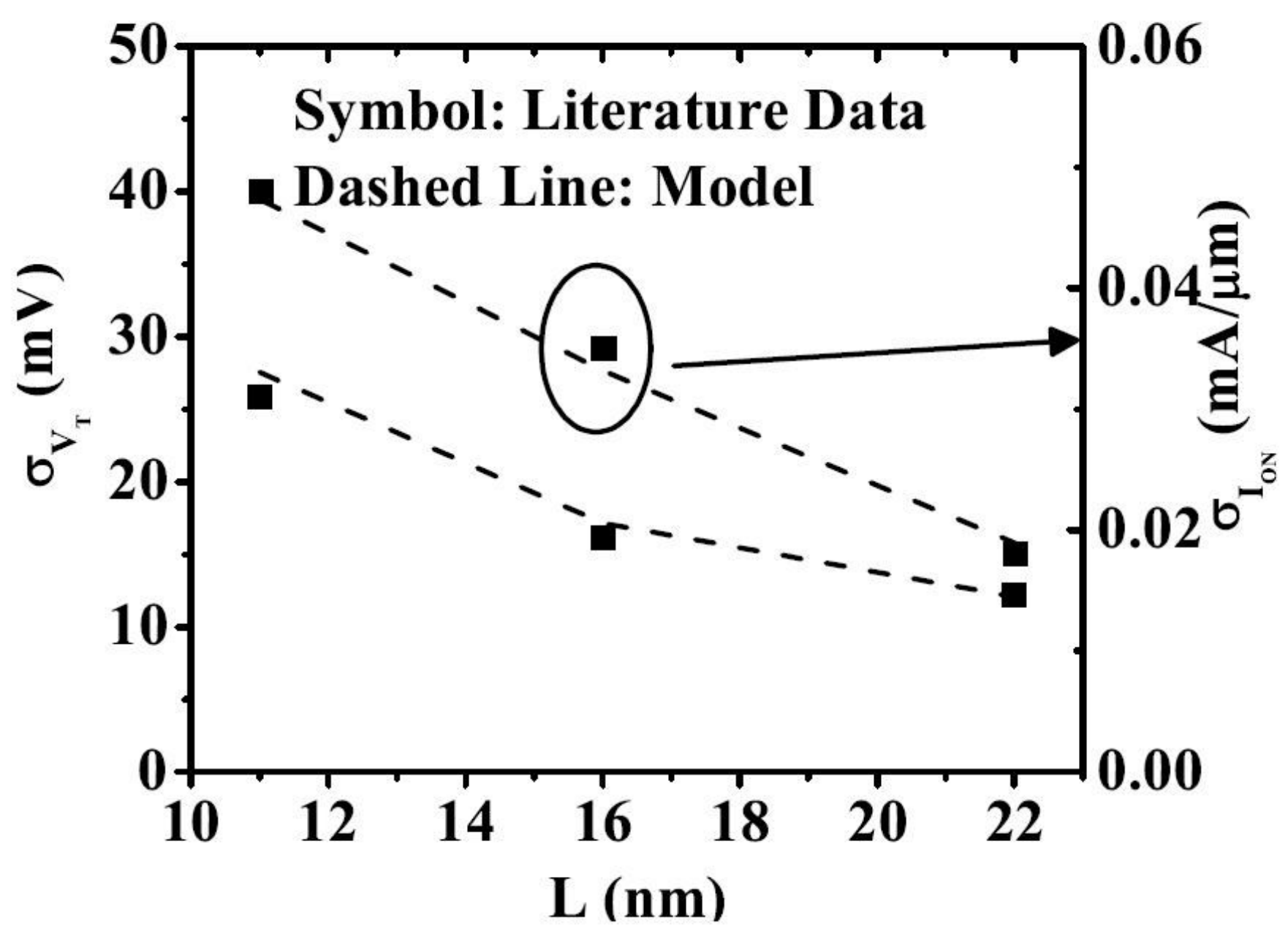

Figure 7

Verification of the model predicted local ON-current variability result due to LER with atomistic simulation results for a UTB-FD-SOI MOSFET [32]. 